\title{
TRATAMIENTO LAPAROSCÓPICO DE LA HERNIA INGUINAL POR TÉCNICA TRANSABDOMINAL PREPERITONEAL. ANÁLISIS RETROSPECTIVO DE UNA SERIE DE CASOS
}

\author{
JOSÉ LUIS LEYBA \\ SALVADOR NAVARRETE AULESTIA² \\ SALVADOR NAVARRETE LLOPIS ${ }^{2}$ \\ LILIHER GONZALEZ ${ }^{3}$
}

\section{LAPAROSCOPIC TREATMENT OF INGUINAL HERNIAS BY TRANSABDOMINAL PREPERITONEAL TECHNIQUE. RETROSPECTIVE ANALYSIS OF A CASE SERIES}

\section{RESUMEN}

Introducción: El objetivo del presente trabajo es reportar y analizar nuestra experiencia con la técnica endoscópica transabdominal preperitoneal (TAPP) en el tratamiento de la hernia inguinal. Métodos: Estudio retrospectivo mediante la revisión de nuestra base de datos electrónica y las historias clínicas en físico de la Clínica Santa Sofía, de los pacientes con diagnóstico de hernia inguinal tratados por los autores en los últimos 8 años. Se incluyeron para el análisis los casos con abordaje laparoscópico, recolectándose sexo, edad, diagnóstico pre e intraoperatorio, procedimientos adicionales, tiempo quirúrgico, complicaciones perioperatorias, tiempo de hospitalización, y morbilidad. Resultados: Se recolectaron 158 intervenciones de hernias inguinales en 102 pacientes, 89 hombres y 13 mujeres, de las cuales 135 (85,4\%) fueron por abordaje laparoscópico tipo TAPP. Mediante la laparoscopia se modificó el diagnóstico preoperatorio en 17 pacientes (16.6\%). El tiempo quirúrgico promedio fue de 50,5 11 1,6 minutos, y se presentaron 3 complicaciones perioperatorias y 4 tardías, para una morbilidad total de 5,1\%. La estancia hospitalaria fue de $1 \pm 0,08$ días y el tiempo de seguimiento promedio fue de 7,5 \pm 15,4 meses, detectándose 3 recidivas (2,2\%) en ese período. Cinco pacientes presentaron dolor crónico posoperatorio, ninguno debilitante, que cedió gradualmente en todos los casos utilizando analgésicos comunes. Conclusiones: La técnica TAPP es una alternativa válida en el tratamiento de la hernia inguinal. Sus principales ventajas son las de optimizar el diagnóstico, permitiendo la reparación en un solo tiempo de hernias no diagnosticadas clínicamente, y su baja incidencia de dolor posoperatorio crónico.

Palabras clave: Hernia inguinal, laparoscopia, transabdominal, preperitoneal, serie de casos.

. Cirujano general. Clínica Santa Sofía. Caracas. Venezuela. Correo-e: jlleyba@yahoo.es.

2. Cirujano general. Clínica Santa Sofía. Caracas. Venezuela.

3. Residente-Cirugía laparoscópica avanzada Clínica Santa Sofia.

\section{ABSTRACT}

Introduction: Currently the Lichtenstein technique is the most used worldwide for inguinal hernia repair, however, the laparoscopic approach has been gaining ground in recent years. The objective of the present work is to present and analyze our experience with the transabdominal preperitoneal (TAPP) technique for the treatment of this pathology. Methods: A retrospective study by the revision of our electronic database and the Santa Sofía clinic physical medical records, of the patients with groin hernia diagnosis treated by the authors in the last 8 years. The cases with the laparoscopic approach were included, collecting sex, age, pre and intraoperative diagnosis, additional procedures, surgical time, perioperative complications, hospital stay, and morbidity. Results: A 158 inguinal hernia repairs were collected in 102 patients, 87 males, and 13 females, of which 135 (85.4\%) were through laparoscopic approach TAPP type. By means of laparoscopy, the preoperative diagnosis was modified in 17 patients (16.6\%). Mean surgical time was $50.5 \pm 11.6$ minutes, and 3 perioperative complications and 4 late complications were observed, for total morbidity of $5.1 \%$. Hospital stay was $1 \pm 0.08$ days, and mean follow-up was $7.5 \pm 15.4$ months, detecting 3 recurrences (2.2\%) at that time. Five patients presented chronic postoperative pain, none debilitating, which gradually yielded in all cases by common pain relievers. Conclusions: The TAPP technique is a valid alternative for the treatment of an inguinal hernia. Its main advantages are to optimize the diagnosis, allowing the repair of clinically undiagnosed hernias in one surgical time, and its low incidence of chronic postoperative pain.

Key words: Inguinal hernia, laparoscopy, transabdominal, preperitoneal, series of cases. 


\section{INTRODUCCIÓN}

Debido a su alta frecuencia y a su impacto en incapacidad laboral y social, las hernias inguinales representan una de las patologías quirúrgicas mas importantes del mundo. (1)

Desde el punto de vista de técnica quirúrgica, la aparición de materiales protésicos que permitieron las reparaciones libres de tensión, lograron no solo simplificar el procedimiento, sino también aumentar significativamente las tasas de éxito. ${ }^{(2,3)}$

El abordaje endoscópico de la hernia inguinal, bien sea totalmente extraperitoneal (TEP) o transabdominal preperitoneal (TAPP), no ha logrado sin embargo una aceptación masiva por parte de la comunidad quirúrgica. ${ }^{(3-6)}$ Esto se debe entre otras cosas a su mayor complejidad técnica con una curva de aprendizaje larga, a la falta de familiaridad de los cirujanos con la visión posterior de la anatomía inguino-crural, así como también a los costos operatorios mas elevados en relación al procedimiento convencional.

A pesar de estos obstáculos, el abordaje endoscópico posee varias ventajas, como la posibilidad de evaluar toda la anatomía del orificio miopectíneo, optimizando así el diagnóstico; y en cuanto a la reparación de la misma, permite colocar la prótesis en un plano anatómico mas amplio y profundo, con lo cual cabe esperar una mayor cobertura del defecto herniario y una menor tasa de recidivas.

En el presente trabajo, reportamos nuestra experiencia con el abordaje laparoscópico de las hernias inguinales, utilizando la técnica TAPP.

\section{MÉtodos}

Se trata de un estudio retrospectivo de los pacientes con diagnóstico de hernia inguinal sometidos a reparación laparoscópica tipo TAPP por nuestro grupo de trabajo (Dres. Leyba, Navarrete Aulestia, y Navarrete Llopis).

Se revisaron las historias de nuestra base de datos electrónica (Lotus Notes 8.5. IBM Corporation ${ }^{\circledR}$ ), así como las historias en físico de la clínica Santa Sofía, de los pacientes con diagnóstico de hernia inguinal intervenidos por nosotros desde enero de 2012 hasta septiembre de 2020 .

Mediante el diseño de una hoja de recolección de datos Microsoft Excel, se recopilaron de los casos intervenidos mediante abordaje laparoscópico la siguiente información: sexo, edad, diagnóstico pre e intraoperatorio, procedimientos adicionales, tiempo quirúrgico, complicaciones perioperatorias, tiempo de hospitalización, y morbilidad.

\section{Técnica quirürgica}

Con el paciente en decúbito dorsal, con sonda vesical de Foley y bajo anestesia general, el cirujano se ubica en la cabecera y el camarógrafo del lado contralateral al sitio de la hernia.

Se creó el neumoperitoneo por técnica Hasson en la línea media a $2 \mathrm{~cm}$ por encima de la cicatriz umbilical hasta una presión de 14 mm Hg. En los casos con diagnóstico preoperatorio de hernia umbilical, el mismo se realizó a nivel de la cicatriz umbilical. Mediante una óptica de $10 \mathrm{~mm}$ y $30^{\circ}$ y bajo visión directa se colocaron dos portales de trabajo de $5 \mathrm{~mm}$ a nivel de ambas líneas medioclaviculares a $2 \mathrm{~cm}$ por debajo del reborde costal.

Se comenzó con la creación del espacio preperitoneal mediante sección del peritoneo parietal a $2 \mathrm{~cm}$ por dentro y arriba de la espina ilíaca anterosuperior en dirección horizontal hasta la línea media. Mediante electrocoagulación monopolar y tijera fría se disecó este espacio en dirección caudal, y con los vasos epigástricos como referencia se continuó la disección hasta visualizar el orificio inguinal profundo y la pared inguinal posterior (lateral y medial respectivamente a los vasos epigástricos). Se identificó la rama horizontal del pubis y el ligamento de Cooper, disecando parcialmente el espacio de Retzius por debajo de ambos de manera de crearle lugar al borde inferior de la malla. Se procedió a reducir todo el saco herniario mediante tracción del mismo y sección de sus adherencias, liberando el peritoneo de los componentes del cordón espermático o del ligamento redondo del útero. La disección del espacio preperitoneal se extendió lateralmente a $5 \mathrm{~cm}$ del anillo inguinal profundo.

Un vez constatada la hemostasia se introdujo a través del portal de $10 \mathrm{~mm}$, una malla de polipropileno rectangular, con una pestaña inferior interna y una muesca superior externa que sirven para orientar la misma dentro del abdomen. El tamaño de la misma depende del defecto herniario, de manera que debe cubrirlo con un margen de al menos $5 \mathrm{~cm}$ en todas las direcciones, lo cual se logra en la mayoría de los casos usando 10 por $15 \mathrm{~cm}$.

Se fijó la pestaña de malla mediante un tacker de titanio al ligamento de Cooper y se dispuso la misma de modo de cubrir toda la pared posterior y el orificio profundo de la región inguinal.

Finalmente se cubrió la malla con el peritoneo parietal, suturando el colgajo que se creó con la disección del mismo y el saco herniario reducido al sitio donde se seccionó inicialmente, mediante una sutura continua de Vicryl 2-0. Se retiraron los portales bajo visión directa, evacuando el neumoperitoneo y suturando la fascia del portal de $10 \mathrm{~mm}$.

\section{Resultados}

Durante el período revisado se operaron 158 hernias inguinales en 102 pacientes, 89 hombres y 13 mujeres, de las cuales 135 (85,4\%) fueron por abordaje laparoscópico tipo TAPP y 23 (14,5\%) mediante inguinotomía por técnica de Lichtenstein.

Se incluyen en el análisis solo los casos laparoscópicos ( $n=135)$, de los cuales 30 (22,2\%) fueron hernias bilaterales, y 6 (4.4\%) fueron casos recidivados.

El tiempo quirúrgico promedio fue de 50,5 1 1 1,6 minutos (40 a 100 minutos) y se presentaron tres (2,2\%) complicaciones perioperatorias: una lesión de la vena espermática, una sección del conducto deferente, ambas tratadas con ligadura de las respectivas estructuras, y una hemorragia con anemia aguda en las primeras horas de posoperatorio, debido a lesión de los vasos epigástricos, que requirió laparotomía para control del sangrado 
más transfusión de hemoderivados.

Un caso se convirtió a inguinotomía debido a la imposibilidad de crear el espacio preperitoneal en una paciente con hernia recidivada.

En 17 pacientes (16,6\%) la laparoscopia modificó el diagnóstico preoperatorio: en 8 casos con diagnóstico inicial de hernia unilateral se demostró en la laparoscopia la presencia de hernia inguinal bilateral, en 5 casos con diagnóstico previo de hernia bilateral se obtuvo como hallazgo hernia unilateral a la exploración laparoscópica, y en 4 pacientes, todas mujeres, se diagnosticaron hernias crurales concomitantes. Se realizaron 23 procedimientos adicionales, siendo el mas frecuente la cura operatoria de hernia umbilical. (Tabla 1)

Tabla 1. Procedimientos adicionales en la reparación laparoscópica de hernias inguinales por técnica TAPP

Cirugía

Reparación de hernia umbilical $\mathrm{n}$

Colecistectomía laparoscópica

Reparación laparoscópica de hernia crural

Funduplicatura laparoscópica

Exéresis de tumor de partes blandas

Otras: histerectomía vaginal, varicocele, esterilización quirúrgica laparoscópica, cistocele

\begin{tabular}{ll}
\hline Total & 36 \\
\hline
\end{tabular}

La estancia hospitalaria fue de 1 $\pm 0,08$ días ( 1 a 2 días), y se presentaron 4 complicaciones tardías (2,9\%): un seroma en la región crural, un hematoma inguinal, y dos hidroceles. Esto implica una morbilidad total (temprana y tardía) de 5. 1\%. (Tabla 2)

Cinco pacientes (3,7\%) presentaron dolor crónico posoperatorio, ninguno debilitante, que cedió gradualmente en todos los casos utilizando analgésicos comunes

El tiempo de seguimiento promedio fue de 7,5 $\pm 15,4$ meses ( 1 a 72 meses) y se detectaron 3 recidivas (2,2\%).

Tabla 2. Complicaciones luego de reparación laparoscópica de hernias inguinales por técnica TAPP

Complicación n $\%$

Perioperatorias

Lesión de vena espermática

Lesión de conducto deferente

$1 \quad 0,7$

Hemorragia intraabdominal por lesión de $1 \quad 0,7$ vasos epigástricos

\begin{tabular}{lll}
\hline Subtotal & 3 & 2,2 \\
\hline Tardias & & \\
Seroma crural & 1 & 0,7 \\
Hematoma inguinal & 1 & 0,7 \\
Hidrocele & 2 & 1,4 \\
\hline Subtotal & 4 & 2,9 \\
\hline Total & 7 & 5,1 \\
\hline
\end{tabular}

\section{Discusıón}

No existe una técnica quirúrgica única para el tratamiento de las hernias inguinales, por lo que la elección de la misma depende de varios factores: características del paciente, tipo de hernia, experiencia del cirujano, y recursos del centro de salud.

Actualmente el abordaje anterior con malla protésica (Lichtenstein) es el más utilizado a nivel mundial, mientras el endoscópico, TEP O TAPP, tiene un alcance variable incluso en centros especializados donde todos los recursos están disponibles. ${ }^{(3-9)}$

En ambas técnicas endoscópicas (TEP y TAPP) se coloca una malla protésica en el espacio preperitoneal, pero solo la última es verdaderamente laparoscópica, ya que en el TEP, dicho espacio se diseca vía anterior, con o sin la ayuda de un dispositivo en forma de balón, pero sin incidir el peritoneo parietal.

La técnica TAPP fue descrita por primera vez en 1992 por Maurice Arregui (10) y a pesar de que nuestra experiencia con cirugía laparoscópica avanzada empezó a finales de los años 90 (11,12), es solo hasta el 2012 cuando comenzamos a tratar las hernias inguinales mediante un abordaje laparoscópico.

El abordaje "abierto" o por inguinotomía mediante técnica de Lichtenstein era nuestro tratamiento habitual, y sus buenos resultados, junto a su relativa simpleza, provocó una resistencia a adoptar una técnica más compleja cuyos beneficios nos parecieron al principio bastante relativos

Sin embargo, hay una serie de condiciones en las que el abordaje endoscópico puede proporcionar algunas ventajas sobre la inguinotomía: 1. Hernias recidivadas cuando el abordaje inicial fue anterior, para evitar la distorsión anatómica causada por la cicatrización de la herida previa; 2. Sexo femenino, por la posibilidad de una hernia crural; 3. Hernia bilateral, sobretodo si no ha sido diagnosticada clínicamente; y 4. Obesidad, al reducir el tamaño de la herida y minimizar así la infección de la misma.

Además, diferentes estudios han demostrado que el abordaje endoscópico es costo/efectivo, y proporciona una recuperación/reincorporación a actividades cotidianas más rápida, así como una menor incidencia de dolor posoperatorio crónico. ${ }^{|3,13-15|}$

Por otro lado, las técnicas endoscópicas (TEP y TAPP) tienen ventajas y desventajas propias, por ejemplo, en el TAPP es mas fácil identificar la anatomía, se reducen las probabilidades de lesión vascular, y es preferible en las hernias bilaterales, pero como contraparte, las lesiones intraabdominales son más frecuentes y es necesario cerrar el peritoneo parietal, Io cual puede ser un tiempo laborioso. (3)

Hasta ahora la literatura ha fallado en demostrar una clara superioridad de un abordaje endoscópico sobre otro, siendo inconsistentes los estudios comparativos sobre ambas técnicas en cuanto a tiempo operatorio, complicaciones, y recurrencias. ${ }^{(16-17)}$

En nuestra experiencia, decidimos elegir el TAAP debido a una anatomía mas familiar, buscando una curva de aprendizaje mas corta. 
Nuestros resultados en cuanto a tiempo quirúrgico y complicaciones son bastante similares a los reportados en la literatura, con una baja tasa de recidivas, aunque reconocemos que la naturaleza retrospectiva del estudio no es la mejor para determinar esto último, siendo una limitante importante de nuestro trabajo. Debido a que la tasa exacta de recurrencia es dificil de estimar, se usa entonces la de reoperaciones para tener una aproximación. Específicamente en el TAPP, las grandes series comparativas reportan recurrencias entre 0,77 y $2,3 \%$. (3)

Un aspecto significativo de nuestra casuística es como la laparoscopia cambió el diagnóstico preoperatorio en un número importante de pacientes (16,6\%), detectando hernias contralaterales o crurales no diagnosticadas, y descartando bilateralidad en algunos casos.

En cuanto a la estadía hospitalaria, si bien esta técnica puede realizarse de manera ambulatoria, en nuestra experiencia hemos dejado hospitalizado siempre al paciente por al menos 24 horas, debido a una natural precaución en nuestra curva de aprendizaje, la cual ha sido estimada entre 50 y 100 casos en estudios previos. ${ }^{(3,20)}$

Uno de los aspectos mas importantes luego de una reparación de hernia inguinal es el dolor crónico posoperatorio. Este se define como aquel que dura mas de tres meses luego de la intervención, y aunque en la mayoría de los pacientes el mismo cede paulatinamente, en algunos casos puede persistir y ser debilitante, afectando las actividades cotidianas. Estos últimos se estiman entre 2-5\% luego del abordaje tipo TAPP, siendo una de las principales ventajas de esta técnica sobre el procedimiento de Lichtenstein. ${ }^{(3,13-15)}$ En nuestra serie la presencia de dolor crónico fue del 3,7\%, sin casos severos o debilitantes, cediendo paulatinamente en todos los pacientes mediante la administración de analgésicos comunes.

De este modo concluimos que la técnica TAPP es una alternativa válida en el tratamiento de la hernia inguinal. Sus principales ventajas son las de optimizar el diagnóstico, permitiendo la reparación en un solo tiempo de hernias no diagnosticadas clínicamente, su baja incidencia de dolor posoperatorio crónico, y la rápida reincorporación del paciente a sus actividades habituales. Algunas condiciones del paciente (obesidad, hernia recidivada, sexo femenino) pueden ser muy atractivas para seleccionar este abordaje, sin embargo, la decisión final dependerá en gran medida del dominio de las diferentes técnicas por parte del equipo quirúrgico.

\section{Conflicto de intereses}

Los autores declaran no tener conflicto de intereses.

\section{Referencias}

1. Kingsnorth A, LeBlanc K. Hernias: inguinal and incisional. Lancet. 2003; 362:1561-1571
2. Lichtenstein IL, Shulman AG, Amid PK, Montllor MM. The tension-free hernioplasty. Am J Surg. 1989; 157(2): 188-193.

3. HerniaSurge Group. International guidelines for groin hernia management. Hernia. 2018; 22:1-165

4. Tran H, Tran K, Turingan I, Zajkowska M, Lam V, Hawthorne W. Single-incision laparoscopic inguinal herniorraphy with telescopic extraperitoneal dissection: technical aspects and potential benefits. Hernia. 2015; 19:407-416.

5. Tschuor C, Metzger J, Clavien PA, Vonlanthen R, Lehmann K. Inguinal hernia repair in Switzerland. Hernia. 2015; 19:741-745.

6. Swedish Hernia Registry. Disponible en http://mmw.svensktbrackregister. se/images/stories/doc/verksamhetsberattelser/rapport 16_170508. pdf.

7. Simons MP, de Lange D, Beets GL, et al. The 'Inguinal Hernia' guideline of the Association of Surgeons of the Netherlands. Nederlands tijdschrift voor geneeskunde. 2003; 147:2111-7.

8. Bittner R, Montgomery MA, Arregui E, et al. Update of guidelines on laparoscopic (TAPP) and endoscopic (TEP) treatment of inguinal hernia (International Endohernia Society). Surg Endosc. 2015; 29:289-321.

9. Bittner R, Schwarz J. Primary unilateral not complicated inguinal hernia: our choice of TAPP, why, results and review of literatura. Hernia. 2019; 23:417-428

10. Arregui ME, Davis CJ, Yucel O. Laparoscopic mesh repair in inguinal hernia using a preperitoneal approach: a preliminary report. Surg Laparosc Endosc. 1992; 2:53-58

11. Navarrete S, Leyba JL, Dehollain A. Tratamiento quirúrgico por laparoscopia de la acalasia: comunicación preliminary. Gac Med Caracas. 2000; 108:196-199

12. Navarrete S, Leyba JL, Dehollain A, Li S. What is the real value of antireflux surgery? JSLS. 2002; 6:139-142.

13. Schmedt CG, Sauerland S, Bittner R. Comparison of endoscopic procedures vs Lichtenstein and other open mesh techniques for inguinal hernia repair: a meta-analysis of randomized controlled trials. Surg Endosc. 2005; 19:188-199

14. Lyu Y, Chenh Y, Wang B, Du W, Xu Y. Comparision of endoscopic surgery and Lichtenstein repair for treatment of inguinal hernias. A network meta-analysis. Medicine. 2020; 99:e19134

15. Bullen NL, Massey LH, Antoniou SA, Smart NJ, Fortelny RH. Open versus laparoscopic mesh repair of primary unilateral uncomplicated inguinal hernia: a systematic review with meta-analysis and trial sequential analysis. Hernia. 2019; 23:461-472

16. McCormack K, Wake BL, Fraser C, Vale L, Perez J, Grant A. Transabdominal pre-peritoneal (TAPP) versus totally extraperitoneal (TEP) laparoscopic techniques for inguinal hernia repair: a systematic review. Hernia. 2005; 9:109-114.

17. Bracale U, Melillo P, Pignata G, Di Salvo E, Rovani M, Merola G, y col. Which is the best laparoscopic approach for inguinal hernia repair: TEP or TAPP? A systematic review of the literature with a network meta-analysis. Surg Endosc. 2012; 26:3355-3366

18. Antoniou SA, Antoniou GA, Bartsch DK, Fendrich V, Koch $\bigcirc 0$, Pointner $R$, y col. Transabdominal preperitoneal versus totally extraperitoneal repair of inguinal hernia: a meta-analysis of randomized studies. Am 
J Surg. 2013; 206:el

19. Wei FX, Zhang YC, Han W, Zhang YL, Shao Y, Ni R. Transabdominal preperitoneal (TAPP) versus totally extraperitoneal (TEP) for laparoscopic hernia repair: a meta-analysis. Surg Laparosc Endosc Percutan Tech. 2015; 25:375-383

20. Bracale U, Merola G, Sciuto A, Cavallaro G, Andreuccetti J, Pignata G.J. Achieving the learning curve in laparoscopic inguinal hernia repair by Tapp: a quality improvment study. Invest Surg. 20 19; 32:738-745. 